\title{
Study on All-Phrase FFT Spectrum Analysis Technology in 25Hz Series Track Circuit Receiver
}

\author{
Shen Tuo ${ }^{1, a^{*}}$, Wei Lexiang ${ }^{1, b}$, Zeng Xiaoqing ${ }^{1, c}$ \\ ${ }^{1}$ School of Transportation Engineering, TongjiUniversity, Shanghai, China \\ ${ }^{a}$ st8250@163.com, bwlx_1022@qq.com, ${ }^{c}$ zengxq@tongji.edu.cn
}

Keywords:25Hz, Track Circuit, APFFT, Electronic receiver

\begin{abstract}
This essay, aims at measuring general track power supply and a local one, manages to apply all-phrase FFT spectrum analysis technology to $25 \mathrm{~Hz}$ series track circuit receiver. By taking advantage of the phrase invariance character of the APFFT, which provides us with precise phrase difference under asynchronous sampling without adjustment, we greatly increase the efficiency of a MPU-based $25 \mathrm{~Hz}$ Series track circuit receiver. We also give a simulation under MATLAB to verify the character.
\end{abstract}

\section{Introduction}

The 25Hzseries track circuit, which was developed in the USSR and Japan in 1960s, has been widely applied in electrified railways. Domestic applications starts from 1978 and the $25 \mathrm{~Hz}$ series track circuit has been the major form of track circuit ever since. As the development of electronics technology, especially the application of digital signal processing technology and embedded micro processing unit in track circuits, the $25 \mathrm{~Hz}$ series track circuit is changing in the direction of electronic and digital.

The key of $25 \mathrm{~Hz}$ series track circuit receiving is measuring the phrase difference between general and local power source by applying digital signal processing technology, significantly digital mutual correlation method and FFT. The two method, although can be applied to phase difference measuring, have following disadvantages: 1) computing works of themare heavy, and thus demand high speed digital signal processing units; 2) their precisions are not satisfactory ${ }^{[1]}$. Because of those disadvantages, a MPU-based $25 \mathrm{~Hz}$ series track circuit receiver, the computing capacity of which are typically limited, is not appropriate for FFT method. In order to reach a satisfying precision, a new algorithm is required.

This essay discussed the application of All-Phrase FFT(APFFT) spectrum analysis technology in measuring the phrase difference between the power sources. The APFFT, which provides us with precise phrase difference under asynchronous sampling without adjustment, requires less computing capacity than the origin method.

\section{Theory of APFFT Spectrum Analysis}

The APFFT Spectrum Analysis method was first put forward and proved superior then the origin FFT due to its character to restraining spectrum leakage in reference [3]. Reference [2] pointed out the two drawbacks of original FFT: that only when the sampling frequency is divisible by the FFT frequency can the result be accurate, otherwise adjustment is needed, and that the spectrum leakage of original FFT may be harmful for the measuring, was the reason why the precision of it was 
disappointing. Also, the phrase invariance character of APFFT, which need no frequency estimation or frequency adjustment to measure the phrase of peak spectral, was proved in reference [2].

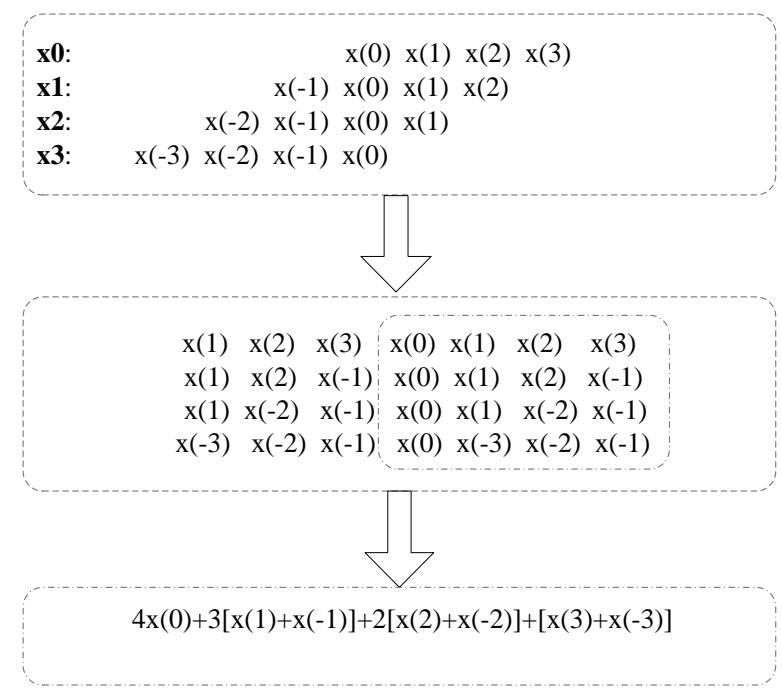

Figure 1 the data processing of APFFT(set $\mathrm{N}=4$ as an example)

As is shown in Fig.1, the APFFT processes as follows:

1) Input a lengths $(2 \mathrm{~N}-1)$ data vector;

2) Set the center of sample as origin, and continue the $(2 \mathrm{~N}-1)$ data whose lengths is $\mathrm{N}$ periodically;

3) Sum the periodically continued vectors up, and get all-phrase data vector;

4) Use FFT algorithm on the all-phrase data vector and analysis the phrase information from the spectrum analysis result.

Suppose the input length of an original FFT as N, and the input data length of an all-phrase FFT would be $(2 \mathrm{~N}-1)$. The computing times of the APFFT would be $N / 2 * \log _{2} N$, withthat of a original FFT as $(\mathrm{N}-1)$. This shows the computing capacity required by the APFFT is much smaller than original FFT, thus greatly increased the computing efficiency of a MPU-based $25 \mathrm{~Hz}$ series track circuit receiver.

\section{The Application of APFFT in Phrase Difference Measuring}

Here we use a frequency invariant signal as an example:

$x(n)=e^{j\left(\omega_{0} n+\varphi_{0}\right)}=e^{j\left(2 \pi \beta n+\varphi_{0}\right)}$

The ADC(Analog-Digital Converter) would collect one and only group of $\mathrm{N}$-dimensioned vectors including the center of sample $x(0)$ with $\mathrm{N}$ vectors in it:

$x_{0}=[x(0), x(1), \ldots, x(N-1)]^{T}$

$x_{1}=[x(-1), x(0), \ldots, x(N-2)]^{T}$

$x_{2}=[x(-2), x(-1), \ldots, x(N-3)]^{T}$

$x_{N-1}=[x(-N+1), x(-N+2), \ldots, x(0)]^{T}$

Then we shift the vectors circularly to set $x(0)$ as the head of the vectors, and we get $\mathrm{N}$ $\mathrm{N}$-dimensioned vectors as follows:

$x_{0}{ }^{\prime}=[x(0), x(1), \ldots, x(N-1)]^{T}$ 


$$
\begin{aligned}
& x_{1}{ }^{\prime}=[x(0), x(1), \ldots, x(-1)]^{T} \\
& x_{2}{ }^{\prime}=[x(0), x(1), \ldots, x(-1)]^{T} \\
& \quad \ldots \ldots \\
& \quad x_{N-1}{ }^{\prime}=[x(0), x(-N+1), \ldots, x(-1)]^{T}
\end{aligned}
$$

Afterwards we calculate the mean vector of the vector group, and that is the all-phrase data vector:

$$
x_{a p}=\frac{1}{N}[N x(0),(N-1) x(1)+x(N-1) \ldots, x(N-1)+(N-1) x(-1)]^{T}(3-4)
$$

According to the shiftable character of DFT, the transform of equation (3-3) and (3-2) will meet the follow equation:

$$
X_{i}{ }^{\prime}(k)=X_{i}(k) \exp \left(j \frac{2 \pi}{N} i k\right) i, k=0,1, \ldots, N-1
$$

Finally we sum the left side of equation (3-5), and we get:

$$
\begin{aligned}
& X_{a p}(k)=\frac{1}{N} \sum_{i=0}^{N-1} X_{i}^{\prime}(k)=\frac{1}{N} \sum_{i=0}^{N-1} X_{i}(k) \exp \left(j \frac{2 \pi}{N} i k\right) \\
& =\frac{1}{N} \sum_{i=0}^{N-1} \sum_{n=0}^{N-1} x(n-i) \exp \left(j \frac{2 \pi}{N} k i\right) \exp \left(-j \frac{2 \pi}{N} k n\right) \\
& =\frac{1}{N^{2}} \sum_{i=0}^{N-1} \sum_{n=0}^{N-1} e^{j \varphi_{0}} e^{j 2 \pi(n-i) \beta / N} \exp \left(j \frac{2 \pi}{N} k i\right) \exp \left(-j \frac{2 \pi}{N} k n\right) \\
& =\frac{e^{j \varphi_{0}}}{N^{2}} \sum_{i=0}^{N-1} e^{j \frac{2 \pi(\beta-k) i}{N}} \sum_{n=0}^{N-1} e^{j \frac{2 \pi(\beta-k) n}{N}} \\
& =\frac{e^{j \varphi_{0}}}{N^{2}} \frac{1-e^{-j 2 \pi(\beta-k)}}{1-e^{-j \frac{2 \pi(\beta-k)}{N}}} \frac{1-e^{j 2 \pi(\beta-k)}}{1-e^{j \frac{2 \pi(\beta-k)}{N}}} \\
& =\frac{e^{j \varphi_{0}}}{N^{2}} \frac{e^{j \pi(\beta-k)}-e^{-j \pi(\beta-k)}}{e^{j \frac{\pi(\beta-k)}{N}}-e^{-j \frac{\pi(\beta-k)}{N}}} \frac{e^{-j \pi(\beta-k)}-e^{j \pi(\beta-k)}}{e^{-j \frac{\pi(\beta-k)}{N}}-e^{j \frac{\pi(\beta-k)}{N}}} \\
& =\frac{e^{j \varphi_{0}}}{N^{2}} \sin ^{2}[\pi(\beta-k)] / \sin ^{2}\left[\frac{\pi(\beta-k)}{N}\right]
\end{aligned}
$$

As is shown above, the phrase of APFFT spectrum is $\varphi_{0}$, which is the center of sample $x(0)$,is uncorrelated with $(\beta-k)$, and that shows the phrase invariance character of APFFT. By using this character, the peak spectral line can be find from the FFT spectrum, and thus the phrase at the center of sample is available.

To get the phrase difference between local and general power source, we proceed the measuring shown above for 2 times to get the phrase of local and general power source.

\section{Simulation and Comparison}

Although the main frequency of the series track circuit is $25 \mathrm{~Hz}$, a small amount of $50 \mathrm{~Hz}$ signal may mix in. In order to make a full consideration, we set the signal source in the simulation as:

$$
x(n)=2 \sin (2 \pi \cdot 25 t)+0.5 \sin (2 \pi \cdot 50 t)
$$


The input of local source is mainly the $25 \mathrm{~Hz}$ power source from the local frequency divider, the phrase of which is $\pi / 2 \mathrm{a}$ after the general source. So the local power source signal shall be as the follows:

$$
x(n)=2 \sin (2 \pi \cdot 25 t+\pi / 2)+0.5 \sin (2 \pi \cdot 50 t)
$$

According to the time-domain sampling theorem, the sampling frequency $f_{m}$ must be great than 2 times top frequency of sample signal $f_{\text {sample }}$. Since $50 \mathrm{~Hz}$ signal is included in the general source, we set the sample frequency as $128 \mathrm{~Hz}$, and choose the order number as 32, 64, 128, 256.

We carried out the measurement by both FFT method and APFFT method, and make a comparison. The spectrums are shown below:
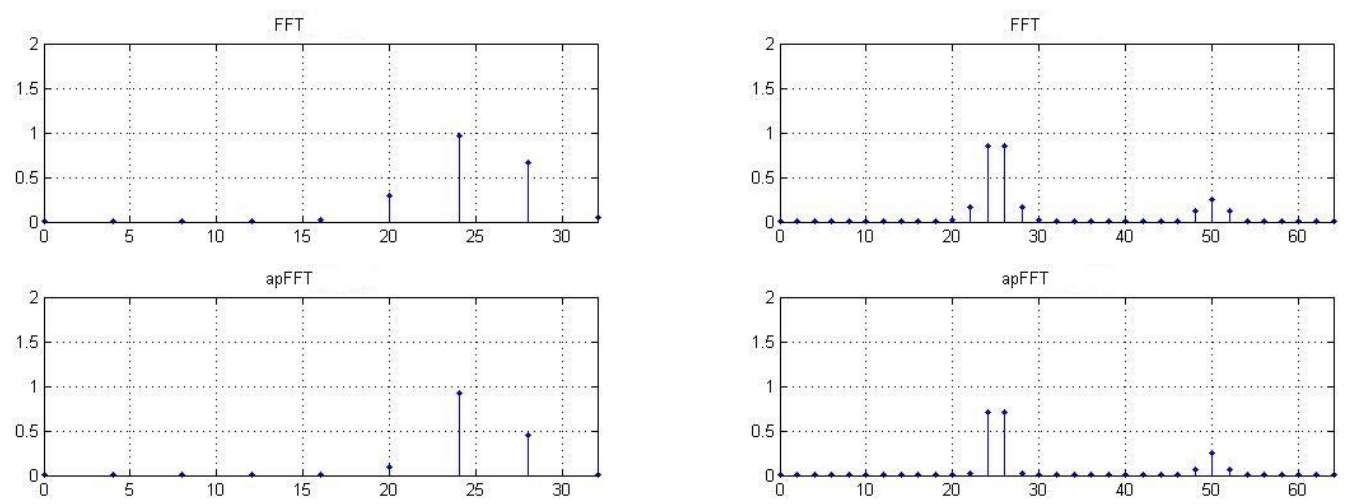

(a) $\mathrm{N}=32, \quad f_{m}=128 \mathrm{~Hz}$

(b) $\mathrm{N}=64, \quad f_{m}=128 \mathrm{~Hz}$
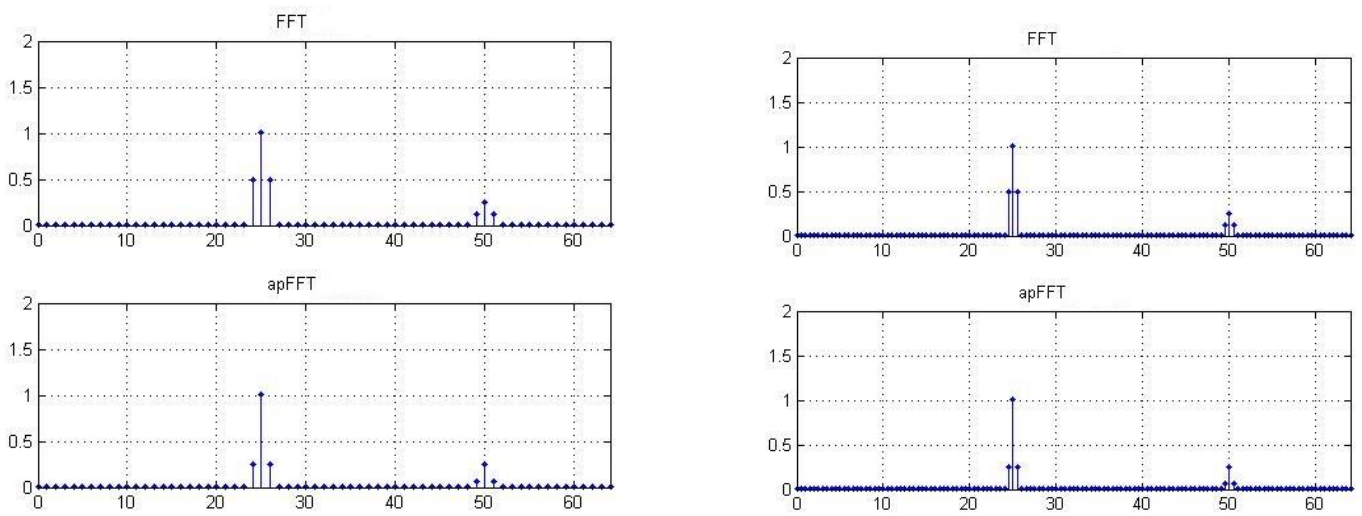

(c) $\mathrm{N}=128, f_{m}=128 \mathrm{~Hz}$

(d) $\mathrm{N}=256, f_{m}=128 \mathrm{~Hz}$

Fig.2 Spectrum of FFT and APFFT under different $\mathrm{N}$

The measuring results of original FFT and APFFT under different computing orders are shown in Fig.2. When $\mathrm{N}=32$, original FFT is unable to give a result due to the lack of samples and spectrum leakage, while the APFFT give a precise result. When $\mathrm{N}=64$, error rate of original FFT is $3.124 \%$ and the APFFT still give a satisfactory measurement. The simulation shows that the original FFT is unable to give an accurate result before $\mathrm{N}$ reaches 256, while the APFFT can make a precise measurement at $\mathrm{N}=32$.

Table 1 measuring result of APFFT and FFT under different $\mathrm{N}$

\begin{tabular}{|c|c|c|c|}
\hline & Original FFT & APFFT & Expected Result \\
\hline $\mathrm{N}=32$ & 2.8024 & $90-1.1474 \mathrm{e}-005$ & 90 \\
\hline $\mathrm{N}=64$ & 87.1879 & $90-1.3276 \mathrm{e}-007$ & 90 \\
\hline $\mathrm{N}=128$ & 89.9998 & $90-1.8220 \mathrm{e}-009$ & 90 \\
\hline $\mathrm{N}=256$ & $90.0000-4.2385 \mathrm{e}-006$ & $90-2.9172 \mathrm{e}-011$ & 90 \\
\hline
\end{tabular}




\section{Summary}

This essay discusses the application of APFFT spectrum analysis technology on $25 \mathrm{~Hz}$ series track circuit. We discussed the theory and algorithm of APFFT, and decide that its character of phrase invariance can overcome the disadvantage of spectrum leakage in original FFT method, thus ensure the precision of the measurement. The computing capacity require by APFFT is smaller than that required by original FFT, and that is beneficial for a MPU-based track circuit receiver.

\section{Acknowledgments}

This work is supported by the Shanghai Committee of Science and Technology, China (12231200103). The author is grateful for the reviewer of initial drafts for their helpful comments and suggestions.

\section{Reference}

[1]WU Hui. 25Hz SeriesTrack Circuit Receiver Design Based On DSP[D]. Beijing: Beijing Jiaotong University, 2007.

[2]Jain, V.K., Collins, William L., Davis, David C. High-Accuracy Analog Measurements via Interpolated FFT[J]. Instrumentation andMeasurement, IEEE Transactions. 1979, 28(2): 113-122.

[3]WANG Zhaohua, HUANG Xiangdong, YANG Wei.Methods For Full-phase FFTPhaseMeasurement[J]. Research and Development of World Technology, 2007,29(4):28-32

[4]WANG Zhaohua, HOU Zhengxi, SU Fei.Allphase FFT Frequency Spectrum Analysis[J].Journal on Communications.2003, 24(11A):16-19.

[5]WANG Zhaohua, HUANG Xiangdong.All Phase Digital Signal Spectral Analysis and Filtering Techniques[M]. Beijing: Publishing House of Electronics Industry,2009.2

[6]WANG Zhaohua, HUANG Xiangdong.Measurement Principles and Applications of All Phase Spectrum Analysis[J] . Journal of Data Acquisition \& Processing2009(6);

[7]Marple, S.L., Jr.. Computing the Discrete-time “Analytic” Signal via FFT[J]. Signal Processing, IEEE Transactions. 1999, 47(9): 2600-2603.

[8]HUANG Xiangdong, WANG Zhaohua.

AllPhaseTime-shiftPhaseDifferenceCorrectingSpectrumMethod [J]. Journal ofTianjin University, 2008,41(7): 815-820

[9]AN Haijun, LI Jianqing, WU Baoying.25HzSeriesTrack Circuit(Third Edition)[M]. Beijing: China Railway Publishing House, 2008.

[10]ZHENG Junli. Signals and Systems[M].Beijing:Higher Education,2000:341-348.

[11]DušanAgrež. Interpolation In the Frequency Domain to Improve Phase Measurement[J]. Advances in Meaurements of Electircal Quantities. 2008,41(2): 151-159. 